\title{
Vectorial form of Ekeland-type variational principle
}

\author{
Kanokwan Sitthithakerngkiet and Somyot Plubtieng*
}

"Correspondence:

Somyotp@nu.ac.th

Department of Mathematics,

Faculty of Science, Naresuan

University, Phitsanulok, 65000,

Thailand

\begin{abstract}
In this paper, we deal with a vectorial form of Ekeland-type variational principle for multivalued bioperator whose domain is a complete metric space and its range is a subset of a locally convex Hausdorff topological space. From this theorem, Caristi-Kirk fixed point theorem for multivalued maps is established in a more general setting and our techniques allow us to improve and to extend their results in (Ansari in J. Math. Anal. Appl. 335: 561-575, 2007; Bednarczuk and Zagrodny in Arch. Math. 93: 577-586, 2009; Bianchi, Kassay and Pini in J. Math. Anal. Appl. 305: 502-512, 2005).
\end{abstract}

MSC: 49K30; 90C29

Keywords: vector variational principle; countably orderable sets; Ekeland's variational principle

\section{Introduction}

It is well known that Ekeland's variational principle (for short, EVP), a very important result first presented by Ekeland [10] in 1974, is as follows:

Theorem 1.1 (Ekeland $[10,11])$ Let $X$ be a complete metric space with a metric d. Let $f$ be a function from $X$ into $(\infty,+\infty$ ] which is proper lower semicontinuous and bounded from below. Then for $u \in X$ and $\lambda>0$, there exists $v \in X$ such that

(i) $f(v) \leq f(u)-\lambda d(u, v)$,

(ii) $f(w)>f(v)-\lambda d(v, w)$ for every $w \neq v$.

It is well known that the primitive EVP is a powerful tool on many applications in optimization, nonlinear analysis, mathematical economy and mathematical programming. Moreover, EVP is equivalent to the Caristis fixed point theorem [5, 8] and nonconvex minimization theorem according to Takahashi [21]. The studies of several forms of Ekeland's variational principle for vector valued functions were obtained by many authors, for instance, Nemeth [20], Tammer [22] and Isac [16, 17].

To begin with, let $X$ be a (real) linear space, $E$ be a (real) topological vector space, $K$ be a cone in $E$ and $e, f: X \rightarrow E$, be two mappings. Under mild conditions of $f$ and $e$, Nemeth [20] got the conclusion that for $\varepsilon>0$ and $y \in X$ there exists a $z \in X$ such that

(i) $-(f(z)-f(y)+\varepsilon e(z-y)) \in K$,

(ii) $-(f(w)-f(z)+\varepsilon e(w-z)) \in K^{c}$; whenever $e(w-z) \neq 0$, where $K^{c}$ is a complement of $K$.

(c) 2012 Sitthithakerngkiet and Plubtieng; licensee Springer. This is an Open Access article distributed under the terms of the Creative Commons Attribution License (http://creativecommons.org/licenses/by/2.0), which permits unrestricted use, distribution, and reproduction in any medium, provided the original work is properly cited. 
Next, let $Y$ be a topological vector space, $K \subseteq X, X$ be a real Banach space and $f: X \rightarrow Y$. Tammer [22] showed that under mild assumptions on $f$ and $K$, for $\varepsilon>0$, there exists an $x \in K$ such that

(i) $f(x)-f\left(x_{\varepsilon}\right)+\varepsilon c^{0} \notin-D \backslash\{0\}, \forall x \in K$,

(ii) $\left\|x_{\varepsilon}-x_{0}\right\| \leq \sqrt{\varepsilon}$,

(iii) $f(x)-f\left(x_{\varepsilon}\right)+\sqrt{\varepsilon}\left\|x-x_{\varepsilon}\right\| c^{0} \notin-D \backslash\{0\}, \forall x \in K$,

where $x_{0}$ is a weakly e-minimal solution of $f$ on $K$ and $c^{0} \in Y \backslash\{0\}$.

On the other hand, by assuming that $(X, d)$ is a complete metric space, $Y$ is a locally convex Hausdorff space and $C \subseteq Y$ is a normal cone, Isac [16] proved that for $\varepsilon>0$ and $x^{\prime \prime} \in X$ satisfying mild conditions, there exists $\lambda>0$ and $x^{\prime} \in X$ such that

(i) $f\left(x^{\prime}\right) \leq C f\left(x^{*}\right)$,

(ii) $d\left(x^{\prime}, x^{*}\right) \leq \lambda$,

(iii) $f(x)-f\left(x^{\prime}\right)+\varepsilon / \lambda d\left(x^{\prime}, x\right) c^{0} \varliminf_{C} 0, \forall x \in X \backslash\left\{x^{\prime}\right\}$,

where $c^{0} \in C \backslash\{0\}$.

Recently, there have been many new formulation cases of EVP in [1-3, 6, 7]. In 2007, a generalization of the Ekeland-type variational principle for vector valued functions in the setting of complete quasi-metric spaces with $\omega$-distance was introduced by Ansari [2]. Let $(X, d)$ be a complete quasi-metric space, $C$ be a proper, closed and convex cone in a locally convex Hausdorff topological vector space $Y$. $F: X \times X \rightarrow Y$ satisfies mild conditions. For every $\varepsilon>0$ and $x_{0} \in X$, there exists $\bar{x} \in X$ such that

(i) $F\left(x_{0}, \bar{x}\right)+\varepsilon \omega\left(x_{0}, \bar{x}\right) e \in-C$,

(ii) $F(\bar{x}, x)+\varepsilon \omega(\bar{x}, x) e \notin-C$, for all $x \in X, x \neq \bar{x}$,

where $e \in \operatorname{int} C$.

By an approach similar to [2], Araya et al. [3] obtained a vectorial version of Ekeland's variational principle related to equilibrium problem. In 2008, Al-Homidan et al. [1] established Ekeland-type variational principles in the setting of quasi-metric spaces with a Q-function. Recently, Bednarczuk and Zagrodny [6] introduced an Ekeland-type vector variational principle for monotonically semicontinuous mappings with perturbations given by a convex bounded subset of directions multiplied by the distance function, and they proved EVP for vector-valued mappings by combining topological and set-theoretic methods. Very recently, Khanh and Quy [19] have proposed a very weak type of generalized distances and used it to weaken the assumptions about lower semicontinuity in the existing versions of Ekeland's variational principle on the complete metric space: to find $v \in X$ such that, for all $x \neq v$

$$
F(v) \nsubseteq F(x)+p(v, x) k_{0}+K,
$$

where $K$ is a convex cone in the Hausdorff locally convex space $Y, F: X \rightarrow 2^{Y}, p$ is a weak $\tau$-function and $k_{0} \in(K-c l K)$.

Motivated by the above mentioned works, we establish a vectorial form of Ekeland-type variational principle for multivalued bioperator whose domain is a complete metric space and its range is a subset of a locally convex Hausdorff topological space by using the set theoretic methods. We also consider Caristi-Kirk fixed point theorem in a more general setting and our techniques allow us to improve and to extend their results in $[2,6,7]$. 


\section{Preliminaries}

This section provides the preliminary terminology and notation used throughout this paper. Let $(X, d)$ be a complete metric space, $Y$ be a locally convex space (i.e., a linear topological space with a local base consisting of convex neighborhoods of the origin, see [15]) and $K$ be a closed convex cone in $Y$. For any $x, y \in Y$ we define

$$
x \leq_{K} y \quad \Leftrightarrow \quad y-x \in K \text {. }
$$

Now, we define the concept of a $\omega$-distance for a metric space which has been introduced by Kada et al. [18].

Definition 2.1 Let $(X, d)$ be a metric space. A function $w: X \times X \rightarrow[0, \infty)$ is called a $w$-distance on $X$ if the following conditions are satisfied:

(i) $w\left(x_{1}, x_{3}\right) \leq w\left(x_{1}, x_{2}\right)+w\left(x_{2}, x_{3}\right)$ for any $x_{1}, x_{2}, x_{3} \in X$;

(ii) for any fixed $x \in X, w(x, \cdot)$ is lower semi continuous;

(iii) for any $\varepsilon>0$, there exists $\delta>0$ such that $w\left(x_{3}, x_{1}\right) \leq \delta$ and $w\left(x_{3}, x_{2}\right) \leq \delta$ implying $d\left(x_{1}, x_{2}\right) \leq \varepsilon$.

Definition 2.2 (See $[9,12])$ Let $f$ be a function from $X$ to $Y$. It is said to be

(i) C-bounded below if there exists $y \in Y$ such that $f(x) \in y+C$, for all $x \in X$;

(ii) $(D, C)$-lower semicontinuous if for all $r \in R,\{x \in X: f(x) \in r D-C\}$ closed;

(iii) (D,C)-upper semicontinuous if for all $r \in R,\{x \in X: f(x) \in r D+C\}$ closed;

(iv) $(D, C)$-continuous if it is both $(D, C)$-lower semicontinuous and $(D, C)$-upper semicontinuous;

(v) (e,C)-lower semicontinuous if for all $r \in R,\{x \in X: f(x) \in r e-C\}$ closed;

(vi) (e,C)-upper semicontinuous if for all $r \in R,\{x \in X: f(x) \in r e+C\}$ closed;

(vii) $(e, C)$-continuous if it is both $(e, C)$-lower semicontinuous and $(e, C)$-upper semicontinuous;

(viii) C-lower semicontinuous if for all $y \in Y,\{x \in X: f(x) \in y-C\}$ closed;

(ix) C-upper semicontinuous if for all $y \in Y,\{x \in X: f(x) \in y+C\}$ closed;

(x) $C$-continuous if it is both $C$-lower semicontinuous and $C$-upper semicontinuous.

Remark 2.3 It is easy to see that the $C$-lower (respectively upper) semicontinuity of $f$ implies the $(e, C)$-lower (respectively upper) semicontinuity.

Definition 2.4 (Holmes [15]) Let $X$ be a linear topological space over the field $\mathbb{R}$.

(1) A sequence $\left\{x_{n}\right\} \subset X$ is bounded if $\lambda_{n} x_{n} \rightarrow \theta$ whenever $\lambda_{n} \rightarrow 0$ in $\mathbb{R}$.

(2) A set $A \subset X$ is bounded if every sequence in $A$ is bounded.

Let $C$ be a convex cone in a linear topological space $Y$ with int $C \neq \emptyset$ and $D$ a convex subset of $C$ such that $0 \notin c l(D+C)$. In order to show the main results, let us give the following definition.

Definition 2.5 A generalized nonlinear scalarization function is defined by

$$
\xi_{(D, C)}(z):=\inf \{r \in \mathbb{R}: z \in r D-C\} \quad \forall z \in Y .
$$


Remark 2.6 If $D=\{e\}$ with $e \in \operatorname{int} C$, then $\xi_{(D, C)}$ Definition 2.5 reduces to the definition of the Gerstewitz function [14]

$$
\xi_{e}(z):=\inf \{r \in \mathbb{R}: z \in r e-C\} \quad \forall z \in Y .
$$

Lemma 2.7 For $r \in \mathbb{R}$, we set $P_{r}=r D-C$. Then the following hold:

(i) If $z \in P_{r}$ for some $r \in \mathbb{R}, z \in P_{\mu}$ for each $\mu>r$.

(ii) For each $z \in Y$, there exists a real number $\lambda$ such that $z \notin P_{\lambda}$.

(iii) Let $z \in Y$. If $z \notin P_{r}$ for some $r \in \mathbb{R}, z \notin P_{\mu}$ for each $\mu<r$.

Proof (i) Let $z \in P_{r}$ for some $r \in \mathbb{R}$ and $\mu>r$. We note that

$$
\mu D-z=(\mu-r) D+r D-z \in C+C \subseteq C .
$$

This implies $z \in P_{\mu}$.

(ii) Assume there exists $z \in Y$ such that for all $\lambda \in \mathbb{R}, z \notin P_{\lambda}$. From (i), we have $z \in \lambda D-C$ for all $\lambda \in \mathbb{R}$. Then we see that

$$
-t D-z \in C \text { and } \quad(1-t) D-z \in C \quad \text { for some } t \in \mathbb{R} \text {. }
$$

Note that

$$
(-t D-z)-((1-t) D-z) \in C+C \subseteq C \text {. }
$$

Since $D$ is convex, we have

$$
0 \in t D+(1-t) D+C \subseteq D+C
$$

which is a contradiction to $0 \notin c l(D+C)$.

(iii) Let $z \in Y$ and if $z \notin P_{r}$ for some $r \in \mathbb{R}$. Assume that for some $\mu<r, z \in P_{\mu}$. From (i), we have $z \in P_{r}$, a contradiction.

Proposition 2.8 The function $\xi: Y \rightarrow \mathbb{R}$ is well defined.

Proof For any $z \in Y$, define

$$
K:=\{r \in \mathbb{R}: z \in r D-C\} .
$$

It is sufficient to show that $K$ is bounded from below.

Assume that for each $r \in \mathbb{R}$, there exists $l \in \mathbb{R}$ such that $l<r$ and $z \in l D-C$.

By Lemma 2.7 (ii), there exists $\mu \in \mathbb{R}$ such that $z \notin \mu D-C$.

By Lemma 2.7 (iii), we have $z \notin \alpha D-C$ for each $\alpha<\mu$, a contradiction. Then $K$ is bounded from below.

Let us recall the basic set-theoretical concepts and tools which are used in the sequel. Let $X$ be a nonempty set and $s \subset X \times X$ a relation. By $x s y$ we mean that $(x, y) \in s$ and we 
write $x s^{*} y$ if and only if there are finite elements $x_{1}, \ldots, x_{n} \in X$ such that

$$
x=x_{1}, x_{1} s x_{2}, \ldots, x_{n-1} s x_{n}, x_{n}=y .
$$

The relation $s^{*}$ is said to be the transitive closure of $s$, and $s=s^{*}$ if $s$ is transitive. We say that the element $x \in X$ is maximal with respect to a relation $s \subset X \times X$ (i.e., $x$ is $s$-maximal) if for every $y \in X$,

$$
x s y \Rightarrow y s^{*} x
$$

Definition 2.9 (See [13]) A set $X$ with a relation $s \subset X \times X$ is countably orderable with respect to $s$ if for every nonempty subset $W \subseteq X$ there exists a well-ordered relation $\mu$ on $W$ such that

$$
v \mu w \Rightarrow v s^{*} w \text { for every } v, w \in W, v \neq w
$$

implies that $W$ is at most countable.

Theorem 2.10 (See [13]) Let $X$ be a countably orderable set by a relation $s \subset X \times X$. Assume that for any sequence $\left(x_{i}\right) \subset X$ satisfying

$$
x_{i} s x_{i+1} \quad \text { for all } i \in \mathbb{N}
$$

there are a subsequence $\left(x_{i_{k}}\right) \subset\left(x_{i}\right)$ and an element $x$ such that

$$
x_{i_{k}} s x \quad \text { for all } k \in \mathbb{N} \text {. }
$$

Then an $s^{*}$-maximal element of $X$ exists.

Moreover, ifs is transitive, then there exists an s-maximal element of $X$.

\section{Main theorem}

In this section, we will present the following vectorial form of an equilibrium version of vector Ekeland's principle in setting complete metric spaces and $\omega$-distance.

Theorem 3.1 Let $X$ be a complete metric space, $\omega: X \times X \rightarrow[0, \infty)$ be a $\omega$-distance on $X$, $Y$ be a locally convex space, $C$ be a closed and convex cone in $Y$ and $D$ be a closed convex and bounded subset of $C$ such that $0 \notin c l(D+C)$. Let $F: X \times X \rightarrow Y$ be a function satisfying the following conditions:

(i) $F(x, x)=0$ for all $x \in X$;

(ii) $F(x, y)+F(y, z) \in F(x, z)+C$ for every $x, y, z \in X$;

(iii) for each $x \in X$, the function $F(x, \cdot): X \mapsto Y$ is $(D, C)$-lower semicontinuous;

(iv) for each fixed $x \in X, F(x, \cdot): X \rightarrow Y$ is $C$-bounded below.

Then for every $x_{0} \in X$, there exists $\bar{x} \in X$ such that

(i) $F\left(x_{0}, \bar{x}\right)+w\left(x_{0}, \bar{x}\right) D \subseteq-C$;

(ii) $F(\bar{x}, x)+w(\bar{x}, x) D \nsubseteq-C$ for all $x \neq \bar{x}$. 
Proof Let $r \subset X \times X$ be a relation defined as follows: For any $x, y \in X$,

$$
x r y \Leftrightarrow F(x, y)+\omega(x, y) D \subseteq-C .
$$

We will first show that $r$ is transitive. Suppose that $u_{1} r u_{2}$ and $u_{2} r u_{3}$. Thus, we have

$$
\begin{aligned}
& F\left(u_{1}, u_{2}\right)+\omega\left(u_{1}, u_{2}\right) D \subseteq-C \text { and } \\
& F\left(u_{2}, u_{3}\right)+\omega\left(u_{2}, u_{3}\right) D \subseteq-C .
\end{aligned}
$$

This implies that

$$
F\left(u_{1}, u_{2}\right)+F\left(u_{2}, u_{3}\right)+\omega\left(u_{1}, u_{2}\right) D+\omega\left(u_{2}, u_{3}\right) D \subseteq-C .
$$

By assumption (ii), we obtain

$$
F\left(u_{1}, u_{3}\right) \in F\left(u_{1}, u_{2}\right)+F\left(u_{2}, u_{3}\right)-C .
$$

Therefore, by the convexity of $D$, we have

$$
\left(\omega\left(u_{1}, u_{2}\right)+\omega\left(u_{2}, u_{3}\right)\right) D=\omega\left(u_{1}, u_{2}\right) D+\omega\left(u_{2}, u_{3}\right) D
$$

Indeed, if $\omega\left(u_{1}, u_{2}\right)+\omega\left(u_{2}, u_{3}\right)=0$, we are done. If $\omega\left(u_{1}, u_{2}\right)+\omega\left(u_{2}, u_{3}\right)>0$, for $d_{1}, d_{2} \in D$, we have

$$
\frac{\omega\left(u_{1}, u_{2}\right)}{\omega\left(u_{1}, u_{2}\right)+\omega\left(u_{2}, u_{3}\right)} d_{1}+\frac{\omega\left(u_{2}, u_{3}\right)}{\omega\left(u_{1}, u_{2}\right)+\omega\left(u_{2}, u_{3}\right)} d_{2} \in D .
$$

So, we have

$$
\omega\left(u_{1}, u_{2}\right) d_{1}+\omega\left(u_{2}, u_{3}\right) d_{2} \in\left(\omega\left(u_{1}, u_{2}\right)+\omega\left(u_{2}, u_{3}\right)\right) D .
$$

Hence $\omega\left(u_{1}, u_{2}\right) D+\omega\left(u_{2}, u_{3}\right) D \subseteq\left(\omega\left(u_{1}, u_{2}\right)+\omega\left(u_{2}, u_{3}\right)\right) D$, which implies that $\left(\omega\left(u_{1}, u_{2}\right)+\right.$ $\left.\omega\left(u_{2}, u_{3}\right)\right) D=\omega\left(u_{1}, u_{2}\right) D+\omega\left(u_{2}, u_{3}\right) D$.

By the definition of $\omega$-distance, $\omega\left(u_{1}, u_{3}\right) \leq \omega\left(u_{1}, u_{2}\right)+\omega\left(u_{2}, u_{3}\right)$. Therefore, there is a real number $\varepsilon>0$ such that

$$
\begin{aligned}
\omega\left(u_{1}, u_{3}\right) D & =\omega\left(u_{1}, u_{2}\right) D+\omega\left(u_{2}, u_{3}\right) D-\varepsilon D \\
& \subseteq \omega\left(u_{1}, u_{2}\right) D+\omega\left(u_{2}, u_{3}\right) D-C .
\end{aligned}
$$

From (3.1), (3.2) and (3.3), we have

$$
F\left(u_{1}, u_{3}\right)+\omega\left(u_{1}, u_{3}\right) D \subseteq-C .
$$

This implies that $u_{1} r u_{3}$.

We define $S: X \rightarrow 2^{X}$ by

$$
S(x)=\{y \in X: F(x, y)+\omega(x, y) D \subseteq-C\} \quad \text { for all } x \in X .
$$


It is easy to see that $x \in S(x)$, and so $S(x)$ is nonempty for all $x \in X$. By assumption (iii), we note that $S(x)$ is a closed set for all $x \in X$. We now show that $S(x)$ is a countably orderable set by a relation $r \subset X \times X$.

Let

$$
V(x):=\inf _{y \in S(x)} \xi_{(D, C)}(F(x, y))
$$

where $\xi_{(D, C)}(z):=\inf \{r \in \mathbb{R}: z \in r D-C\}$ for all $z \in Y$.

Let $W$ be any nonempty subset of A which is well ordered by a relation $s$ satisfying

$$
u s v \Rightarrow u r^{*} v \quad \text { for every } u, v \in W, u \neq v \text {. }
$$

Then, for any $u, v \in W$ with $u \neq v$, we note that

$$
u s v \Rightarrow u r^{*} v \Rightarrow u r v \text { and } S(u) \subseteq S(v)
$$

because $r$ is transitive. Since $u r v, u \neq v$, thus $F(u, v)+\omega(u, v) D \subseteq-C$, which implies that $\xi_{(D, C)}(F(u, v)) \leq-\omega(u, v)<0$. Moreover,

$$
\begin{aligned}
V(u) & =\inf _{y \in S(u)} \xi_{(D, C)}(F(u, y)) \\
& \leq \inf _{y \in S(v)} \xi_{(D, C)}(F(u, y)) \\
& \leq \inf _{y \in S(v)}\left(\xi_{(D, C)}(F(u, v))+\xi_{(D, C)}(F(v, y))\right) \\
& =\xi_{(D, C)}(F(u, v))+\inf _{y \in S(v)} \xi_{(D, C)}(F(v, y)) \\
& <\inf _{y \in S(v)} \xi_{(D, C)}(F(v, y)) \\
& =V(v) .
\end{aligned}
$$

Thus $V(W) \subset \mathbb{R}$ is well ordered by the relation " $<$ " and hence $V(W)$ is at most countable. Since $V$ is one-to-one mapping on $W, W$ is at most countable.

For any $x \in X$, we let $\left(y_{n}\right) \subset S(x)$ with $y_{n} r y_{n+1}$ for all $n \in \mathbb{N}$. We next show that there is an element $y_{0}$ such that $y_{n} r y_{0}$ for all $n \in \mathbb{N}$.

In case $y_{m}=y_{m+1}=y_{m+2}=\cdots$ for some $m \in \mathbb{N}$, we can put $y_{0}:=y_{m}$ and so we have done. Then, it is enough to consider the case $\sum_{i=1}^{\infty} \omega\left(y_{i}, y_{i+1}\right)>0$. Since $y_{n} r y_{n+1}$ for each $n \in \mathbb{N}$, we obtain

$$
F\left(y_{n}, y_{n+1}\right)+\omega\left(y_{n}, y_{n+1}\right) D \subseteq-C .
$$

From (3.4) and assumption (ii), we observe that

$$
\begin{aligned}
F\left(y_{1}, y_{2}\right) \in & F\left(y_{1}, y_{m+1}\right)-F\left(y_{2}, y_{3}\right)-F\left(y_{3}, y_{4}\right)-\cdots-F\left(y_{m}, y_{m+1}\right)+C \\
\subseteq & F\left(y_{1}, y_{m+1}\right)+\left(\omega\left(y_{2}, y_{3}\right) D+C\right)+\left(\omega\left(y_{3}, y_{4}\right) D+C\right)+\cdots \\
& +\left(\omega\left(y_{m}, y_{m+1}\right) D+C\right)+C \\
\subseteq & F\left(y_{1}, y_{m+1}\right)+\sum_{i=2}^{m}\left(\omega\left(y_{i}, y_{i+1}\right) D\right)+C,
\end{aligned}
$$


for all $m \in \mathbb{N}$. Since $F$ is $C$-bounded below, there exists $z \in Y$ such that

$$
F\left(y_{1}, y_{2}\right) \in z+C+\sum_{i=2}^{m}\left(\omega\left(y_{i}, y_{i+1}\right) D\right)
$$

By the convexity of $D$, we have

$$
\sum_{i=1}^{m}\left(\omega\left(y_{i}, y_{i+1}\right) D\right)=\left(\sum_{i=1}^{m} \omega\left(y_{i}, y_{i+1}\right)\right) D
$$

for any $m \in \mathbb{N}$. Therefore, it follows from (3.5) and (3.6) that

$$
F\left(y_{1}, y_{2}\right) \in z+C+\left(\sum_{i=2}^{m} \omega\left(y_{i}, y_{i+1}\right)\right) D .
$$

Since $0 \notin c l(D+C)$, by the Separation theorem, there exists $f^{\prime \prime} \in Y^{\prime \prime}$ such that

$$
\left\langle f^{*}, 0\right\rangle<\inf \left\{\left\langle f^{*}, d+c\right|, \forall d \in D, \forall c \in C\right\}
$$

This implies that $0<\varepsilon<\left\langle f^{*}, d+c\right\rangle=\left\langle f^{*}, d\right\rangle+\left\langle f^{*}, c\right\rangle$ for some $\varepsilon>0$, and for any $d \in D, c \in C$. Hence $\inf _{d \in D}\left\langle f^{*}, d\right\rangle>0$ and $\left\langle f^{*}, c\right\rangle \geq 0$ for any $c \in C$. Hence, for each $m \in \mathbb{N}$, we have

$$
\left\langle f^{*}, F\left(y_{1}, y_{2}\right)\right\rangle=\left\langle f^{*}, z\right\rangle+\left\langle f^{*}, c\right\rangle+\sum_{i=2}^{m} \omega\left(y_{i}, y_{i+1}\right)\left\langle f^{*}, d\right\rangle
$$

for some $c \in C$ and $d \in D$. Since $\left\langle f^{*}, c\right\rangle \geq 0$ for any $c \in C$, it follows that

$$
\left\langle f^{*}, F\left(y_{1}, y_{2}\right)\right\rangle \geq\left\langle f^{*}, z\right\rangle+\sum_{i=2}^{m} \omega\left(y_{i}, y_{i+1}\right) \inf _{d \in D}\left\langle f^{*}, d\right\rangle .
$$

Since $\inf _{d \in D}\left\langle f^{*}, d\right\rangle>0$, we have that $\sum_{i=1}^{m} \omega\left(y_{i}, y_{i+1}\right)$ is bounded above by $\frac{\left\langle f^{n}, F\left(y_{1}, y_{2}\right)\right\rangle-\left\langle f^{n}, z\right\rangle}{\inf _{d \in D}\left\langle f^{n}, d\right\rangle}$. Moreover, $\left(\sum_{i=1}^{m} \omega\left(y_{i}, y_{i+1}\right)\right)$ is a monotone sequence then the series $\sum_{i=1}^{\infty} \omega\left(y_{i}, y_{i+1}\right)$ converges. This implies that $\lim _{i \rightarrow \infty} \omega\left(y_{i}, y_{i+1}\right)=0$. It is easy to see that $\left(y_{n}\right)$ is a Cauchy sequence in $S(x)$. By the completeness of $X$ and closedness of $S(x),\left(y_{n}\right)$ converges to a certain $y_{0} \in S(x)$. Since $r$ is transitive and $y_{n} r y_{n+1}$, then $y_{n} r y_{m}$ for all $m>n$, and so $y_{n} r y_{0}$. This entails that $S(x)$ satisfies the condition in Theorem 2.10. Now, the proof includes applying Theorem 2.10 to show that $S(x)$ has an $r$-maximal element $\bar{x} \in S(x)$. Let us observe that for $x \in X$, any $r$-maximal element of $S(x)$ is an $r$-maximal element of $X$. Hence, (i) holds for $\bar{x}$. Finally, we show that $\bar{x}$ satisfies (ii). Assume that $\bar{x} r z$ for some $z \neq \bar{x}$. Since $r$ is transitive and $\bar{x}$ is $r$-maximal, $z r \bar{x}$. Consequently, $V(\bar{x})>V(z)$ and $V(z)>V(\bar{x})$, a contradiction. Hence $\bar{x}$ satisfies (ii).

Remark 3.2 We see in the proof that we do not use the symmetry condition of the metric. So, the conclusion in Theorem 3.1 still holds if we replace the word "metric space" with "quasi-metric space".

By setting $D=\{\varepsilon e\}$ for all $\varepsilon>0$ in Theorem 3.1, we obtain the following Corollary which is proven by Ansari [2]. 
Corollary 3.3 (Theorem 3.1 in [2]) Let $(X, d)$ be a complete quasi-metric space, $\omega: X \times$ $X \rightarrow[0, \infty)$ a $\omega$-distance on $X, Y$ be a locally convex Hausdorff topological vector space, $C$ be a proper, closed and convex cone in $Y$ with apex at origin and int $C \neq \emptyset$, and $e \in Y$ be $a$ fixed vector such that $e \in \operatorname{int} C$. Let $F: X \times X \rightarrow Y$ be a function satisfying the following:

(i) $F(x, x)=0$, for all $x \in X$;

(ii) $F(x, y)+F(y, z) \in F(x, z)+C$ for all $x, y, z \in X$;

(iii) for each fixed $x \in X$, the function $F(x, \cdot): X \rightarrow Y$ is $(e, C)$-lower semicontinuous and $C$-bounded below.

Then for every $\varepsilon>0$ and for every $x_{0} \in X$, there exists $\bar{x} \in X$ such that

(a) $F\left(x_{0}, \bar{x}\right)+\varepsilon \omega\left(x_{0}, \bar{x}\right) e \in-C$,

(b) $F(\bar{x}, x)+\varepsilon \omega(\bar{x}, x) e \notin-C$, for all $x \in X, x \neq \bar{x}$.

If $F(x, y)=f(y)-f(x)$, where $f: X \rightarrow R$ is lower semicontinuous and bounded below, then we have the following result.

Corollary 3.4 Let $X$ be a complete metric space, $\omega: X \times X \rightarrow[0, \infty)$ be a $\omega$-distance on $X$, $Y$ be a locally convex space, $C$ be a closed and convex cone in $Y$ and $D$ be a closed convex and bounded subset of $C$ such that $0 \notin c l(D+C)$. Let $f: X \rightarrow Y$ be $(D, C)$-lower semicontinuous and $C$-bounded below. Then for every $x_{0} \in X$ there exists $\bar{x} \in X$ such that

(i) $f(\bar{x})+w\left(x_{0}, \bar{x}\right) D \subseteq f\left(x_{0}\right)-C$;

(ii) $f(x)+w(\bar{x}, x) D \nsubseteq f(\bar{x})-C$ for all $x \neq \bar{x}$.

We obtain that Corollary 3.4 is the extension of the following.

Corollary 3.5 Let $X$ be a complete metric space, $\omega: X \times X \rightarrow[0, \infty)$ be a $\omega$-distance on $X$, $Y$ be a locally convex space, $C$ be a closed and convex cone in $Y$ and $D$ be a closed convex and bounded subset of $C$ such that $0 \notin c l(D+C)$. Let $f: X \rightarrow Y$ be $(D, C)$-lower semicontinuous and $C$-bounded below. Then for every $x_{0} \in X$ there exists $\bar{x} \in X$ such that

(i) $\left(f\left(x_{0}\right)-C\right) \cap\left(f(\bar{x})+w\left(x_{0}, \bar{x}\right) D\right) \neq \emptyset$;

(ii) $(f(\bar{x})-C) \cap(f(x)+w(\bar{x}, x) D)=\emptyset$ for all $x \neq \bar{x}$.

Proof By all conditions of Corollary 3.4, we have for every $x_{0} \in X$ there exists $\bar{x} \in X$ such that

$$
\begin{aligned}
& f(\bar{x})+w\left(x_{0}, \bar{x}\right) D \subseteq f\left(x_{0}\right)-C, \\
& f(x)+w(\bar{x}, x) D \nsubseteq f(\bar{x})-C \text { for all } x \neq \bar{x} .
\end{aligned}
$$

From 3.7, we have (i) holds.

If (ii) were not satisfied, we would have $(f(\bar{x})-C) \cap(f(x)+w(\bar{x}, x) D) \neq \emptyset$ for some $x \neq \bar{x}$. Then there are $c_{1} \in C$ and $d_{1} \in D$ such that

$$
f(\bar{x})=f(x)+w(\bar{x}, x) d_{1}+c_{1} .
$$

Since $0 \notin \operatorname{cl}(D+C)$, by the Separation theorem, there exists $y^{*} \in Y^{*}$ such that $0<\varepsilon<$ $\left\langle y^{*}, d+c\right\rangle=\left\langle y^{*}, d\right\rangle+\left\langle y^{*}, c\right\rangle$ for some $\varepsilon>0, d \in D$ and $c \in C$. Hence $\inf _{d \in D}\left\langle y^{*}, d\right\rangle>0$ and $\left\langle y^{*}, c\right\rangle \geq 0$ for any $c \in C$. 
From (3.9), we obtain that

$$
\left\langle y^{*}, f(\bar{x})\right\rangle=\left\langle y^{*}, f(x)+w(\bar{x}, x) d_{1}+c_{1}\right\rangle>\left\langle y^{*}, f(x)\right\rangle
$$

Using the same method of (3.9), we conclude that $\left\langle y^{*} f(\bar{x})\right\rangle\left\langle\left\langle y^{*} f(x)\right\rangle\right.$, a contradiction. Consequently (ii) holds

If we set $Y=\mathbb{R}, C=[0, \infty)$ and $D=\{\varepsilon\}$ for $\varepsilon>0$ in Theorem 3.1, we have the following result which is a well-known Ekeland's variational principle in a more general setting.

Corollary 3.6 Let $X$ be a complete metric space, $\omega: X \times X \rightarrow[0, \infty)$ be a $\omega$-distance on $X, f: X \times X \rightarrow \mathbb{R}$ be a function satisfying the following conditions:

(i) $F(x, x)=0$ for all $x \in X$;

(ii) $F(x, y)+F(y, z) \geq F(x, z)$ for every $x, y, z \in X$;

(iii) for each $x \in X$ the function $F(x, \cdot): X \mapsto \mathbb{R}$ is lower semicontinuous and bounded below.

Then for every $x_{0} \in X$ and $\varepsilon>0$, there exists $\bar{x} \in X$ such that

(i) $F\left(x_{0}, \bar{x}\right)+\varepsilon \omega\left(x_{0}, \bar{x}\right) \leq 0$;

(ii) $F(\bar{x}, x)+\varepsilon \omega(\bar{x}, x)>0$ for all $x \neq \bar{x}$.

Remark 3.7 By setting $w=d$ and $F(x, y)=f(y)-f(x)$, where $f: X \rightarrow R$ is lower semicontinuous and bounded below in Corollary 3.6, we obtain Theorem 1.1 proven by Ekeland $[10,11]$.

The following theorem provides the equivalence between the equilibrium version of Ekeland-type variational principle, the equilibrium problem, Caristi-Kirk type fixed point theorem and Oettli and Théra type theorem

Theorem 3.8 Let $X$ be a complete metric space, $\omega: X \times X \rightarrow[0, \infty)$ be a $\omega$-distance on $X$, $Y$ be a locally convex space, $C$ be a closed and convex cone in $Y$ and $D$ be a closed convex and bounded subset of $C$. Let $T: X \rightarrow 2^{X}$ and $F: X \times X \rightarrow Y$ be a function satisfying the following conditions:

(i) $F(x, x)=0$ for all $x \in X$;

(ii) $F(x, y)+F(y, z) \in F(x, z)+C$ for every $x, y, z \in X$;

(iii) for each $x \in X$, the function $F(x, \cdot): X \mapsto Y$ is $(D, C)$-lower semicontinuous;

(iv) for each fixed $x \in X, F(x, \cdot): x \rightarrow Y$ is C-bounded below;

(v) for each $x \in X$, there is $y \in X$ such that $y \in T x$ and $F(x, y)+\omega(x, y) D \subseteq-C$.

Then $T$ has at least one fixed point, i.e., there exists $x \in X$ such that $x \in T x$.

Proof By assumption (i)-(iv) applied to Theorem 3.1, there exists $\bar{x} \in X$ such that

$$
F(\bar{x}, z)+w(\bar{x}, z) D \nsubseteq-C \quad \text { for all } z \neq \bar{x}
$$

On the other hand by assumption (v), there exists $y \in T(\bar{x})$ such that

$$
F(\bar{x}, y)+w(\bar{x}, y) D \subseteq-C .
$$

Then we see that $\bar{x}=y$, and so $\bar{x} \in T(\bar{x})$, that is, $T$ has at least one fixed point. 
Remark 3.9 We set $F(x, y)=f(y)-f(x), D=\{\varepsilon\}, \varepsilon>0$ and replace $\omega$-distance by ddistance in Theorem 3.8, we obtain Theorem 3.1 in [3] and Theorem 4.1 in [4] (vectorial Caristi-Kirk fixed point theorem).

\section{Competing interests}

The authors declare that they have no competing interests.

\section{Authors' contributions}

The work presented here was carried out in collaboration between all authors. SP designed theorems and methods of the proof and interpreted the results. KS proved the theorems, interpreted the results and wrote the paper. All authors read and approved the final manuscript.

\section{Acknowledgement}

The first author would like to thank the Office of the Higher Education Commission of Thailand for supporting by grant fund under the program Strategic Scholarships for the Join Ph.D. Program Thai Doctoral degree for this research.

Received: 22 April 2012 Accepted: 18 July 2012 Published: 2 August 2012

\section{References}

1. Al-Homidan, S, Ansari, QH, Yao, J-C: Some generalizations of Ekeland-type variational principle with applications to equilibrium problems and fixed point theory. Nonlinear Anal., Theory Methods Appl. 69(1), 126-139 (2008)

2. Ansari, QH: Vectorial form of Ekeland-type variational principle with applications to vector equilibrium problems and fixed point theory. J. Math. Anal. Appl. 334, 561-575 (2007)

3. Araya, Y, Kimura, K, Tanaka, T: Existence of vector equilibrium via Ekeland's variational principle. Taiwan. J. Math. 12, 1991-2000 (2008)

4. Araya, Y: Ekeland's variational principle and its equivalent theorems in vector optimization. J. Math. Anal. Appl. 346, 9-16 (2008)

5. Aubin, JP, Siegel, J: Fixed points and stationary points of dissipative multivalued maps. Proc. Am. Math. Soc. 78(3), 391-398 (1980)

6. Bednarczuk, EM, Zagrodny, D: Vector variational principle. Arch. Math. 93, 577-586 (2009)

7. Bianchi, M, Kassay, G, Pini, R: Existence of equilibria via Ekeland's principle. J. Math. Anal. Appl. 305, 502-512 (2005)

8. Caristi, J: Fixed point theorems for mappings satisfying inwardness conditions. Trans. Am. Math. Soc. 215, 241-251 (1976)

9. Chen, GY, Huang, XX: Ekeland's variational principle for set-valued mappings. Math. Methods Oper. Res. 48, 181-186 (1998)

10. Ekeland, I: On the variational principle. J. Math. Anal. Appl. 47, 324-353 (1974)

11. Ekeland, I: Nonconvex minimization problems. Bull. Am. Math. Soc. 1, $443-474$ (1979)

12. Facchinei, F, Pang, JS: Finite Dimensional Variational Inequalities and Complementarity Problems, I. Springer, New York (2003)

13. Gajek, L, Zagrodny, D: Countably orderable sets and their applications in optimization. Optimization 26, 287-30 (1992)

14. Gerth, C, Weidner, P: Nonconvex separation theorems and applications in vector optimization. J. Optim. Theory Appl. 67, 297-320 (1990)

15. Holmes, RB: Geometric Functional Analysis and Its Applications. Springer, New York (1975)

16. Isac, G: Ekeland's principle and Pareto e-efficiency. In: Tamiz, M (ed.) Multi-objective Programming and Goal Programming, Theory and Applications, Lecture Notes in Economic and Mathematical Systems, vol. 432. Springer, Berlin (1996)

17. Isac, G: Nuclear cones in product spaces, Pareto efficiency and Ekeland-type variational principle in locally convex spaces. Optimization 53, 253-268 (2004)

18. Kada, O, Suzuki, T, Takahashi, W: Nonconvex minimization theorems and fixed point theorems in complete metric spaces. Math. Jpn. 44, 381-391 (1996)

19. Khanh, PQ, Quy, DN: On generalized Ekeland's variational principle and equivalent formulations for set-valued mappings. J. Glob. Optim. 49, 381-396 (2011)

20. Nemeth, AB: A nonconvex vector minimization problem. Nonlinear Anal. 10, 669-678 (1986)

21. Takahashi, W: Existence theorems generalizing fixed point theorems for multivalued mappings. In: Thera, MA, Baillon, JB (eds.), Fixed Point Theory and Applications, Pitman Res. Notes in Math. Ser., vol. 252, pp. 397-406. Longman, Harlow (1991)

22. Tammer, C: A generalization of Ekeland's variational principle. Optimization 25, 129-141 (1992)

doi:10.1186/1687-1812-2012-127

Cite this article as: Sitthithakerngkiet and Plubtieng: Vectorial form of Ekeland-type variational principle. Fixed Point Theory and Applications 2012 2012:127. 